\title{
Probióticos em mulheres com candidíase vulvovaginal: qual a evidência?
}

Lucélia Cristina Pedras Campinho, ${ }^{1}$ Susana Margarida Vilar Santos, ${ }^{1}$ Alexandra Cardoso Azevedo ${ }^{2}$

\section{RESUMO}

A candidíase vulvovaginal constitui uma das formas mais comuns de infeção genital feminina, afetando $75 \%$ das mulheres pelo menos uma vez na vida. Está associada a um desequilíbrio da microflora vaginal por consequente alteração quantitativa e/ou qualitativa dos Lactobacillus e sobrecrescimento de outros comensais da mucosa vaginal, nomeadamente a Candida albicans. Os probióticos, que contêm Lactobacillus, são frequentemente usados no tratamento e prevenção da candidíase vulvovaginal.

O objetivo desta revisão é determinar, à luz da evidência atual, se os probióticos contribuem para prevenção e tratamento de mulheres com candidíase vulvovaginal.

Foi feita uma pesquisa de normas de orientação clínica, meta-análises, revisões sistemáticas e ensaios clínicos aleatorizados, publicados entre março de 2007 e março de 2017, nas línguas portuguesa e inglesa. Na pesquisa foram utilizados os termos MeSH candidiasis vulvovaginal e probiotics. Para a avaliação dos níveis de evidência e atribuição das forças de recomendação utilizou-se a escala Strength of Recommendation Taxonomy.

Da pesquisa efetuada resultaram 22 artigos, dos quais dois foram incluídos. Os restantes foram excluídos por não cumprirem os critérios de inclusão, por repetição ou não estarem acessíveis.

Os artigos incluídos são duas normas de orientação clínica que concluíram que não está recomendado o uso de probióticos no tratamento e/ou prevenção da candidíase vulvovaginal, ambas com força de recomendação A.

Considera-se que são necessários mais estudos nesta área, de elevada qualidade, que suportem a evidência encontrada na formulação das recomendações.

Palavras-chave: Candidíase vulvovaginal; Probióticos.

\section{INTRODUÇÃO}

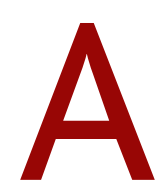
candidíase vulvovaginal (CVV) constitui uma das formas mais comuns de infeção genital feminina, afetando $75 \%$ das mulheres pelo menos uma vez na vida. Ocorre mais frequentemente em mulheres pré-menopausa, mas também em mulheres pós-menopausa sob terapêutica estrogénica. ${ }^{1-2}$ Os fatores de risco para a sua ocorrência são a atividade sexual, a antibioterapia recente, a gravidez e a imunossupressão, associada a infeção pelo vírus da imunodeficiência humana (VIH) mal controlada e a diabetes. ${ }^{2}$

A CVV recorrente ocorre em cerca de 5 a $8 \%$ das mulheres. ${ }^{3}$ Define-se por quatro ou mais episódios de CVV em 12 meses. ${ }^{2}$ Normalmente há um período de remissão dos sintomas entre os episódios. ${ }^{1}$ Apresenta-se frequentemente com sintomas mais severos, na presença

1. Médica Especialista em Medicina Geral e Familiar. ACeS Famalicão.

2. Médica Especialista em Medicina Geral e Familiar. UCSP Cinfães. de Candida não albicans ou perante um hospedeiro imunocomprometido, com infeção por VIH ou diabetes. ${ }^{2}$ É uma condição que causa elevado desconforto e sofrimento, por vezes com impacto psicológico significativo, para além dos custos em saúde.

A CVV está associada a um desequilíbrio da microflora vaginal por consequente alteração quantitativa e/ou qualitativa dos Lactobacillus e sobrecrescimento de outros comensais da mucosa vaginal, nomeadamente a Candida albicans. Outras espécies de Candida estão a emergir como a glabrata, parapsilosis e tropicalis. O principal reservatório de Candida é o reto, sendo comum a colonização vaginal. A evolução da colonização para infeção sintomática envolve múltiplos fatores associados à suscetibilidade e resposta inflamatória do hospedeiro e à virulência da espécie de Candida. ${ }^{2}$ Os Lactobacillus produzem ácido lático, que contribui para a manutenção do $\mathrm{pH}$ fisiológico $<4,5$ e protege a vagi- 
na dos microrganismos que ascendem do trato gastrointestinal. Também produzem peróxido de hidrogénio e bacteriocinas, inibindo a adesão dos microrganismos patogénicos às células epiteliais vaginais. ${ }^{2-3}$

A CVV manifesta-se por leucorreia branca espessa, tipo requeijão, associada a prurido, dor, ardor, eritema e/ou edema vulvovaginal. Pode ocorrer disúria externa e dispareunia. ${ }^{2}$

O diagnóstico da CVV baseia-se na presença de sintomas e sinais. As secreções vaginais apresentam um $\mathrm{pH}$ $<4,5$ e o exame microscópico a fresco mostra leveduras e pseudo-hifas. O teste de Wiffé negativo e o exame microscópico direto com coloração Gram revela células polimorfonucleares, leveduras - esporos e pseudo-hifas. Na presença de CVV recorrente deve efetuar-se colheita de exsudado vaginal para cultura, devido à elevada probabilidade da presença de espécies não albicans. ${ }^{2}$

O tratamento da CVV está recomendado apenas na presença de sintomas. Não está indicado o tratamento do parceiro sexual. Os antifúngicos azóis são o tratamento preferencial, na formulação tópica ou oral, apresentando eficácia semelhante. ${ }^{3} \mathrm{Na}$ CVV recorrente, após terapêutica inicial, está indicada a terapêutica de manutenção durante pelo menos seis meses. Podem ser usadas diferentes classes farmacológicas, com resulta-

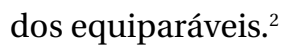

Devido a falhas terapêuticas e microbiológicas do tratamento convencional da CVV, os probióticos foram os primeiros novos agentes introduzidos no tratamento da CVV em 2001. São definidos como "microrganismos vivos que, quando administrados em quantidades adequadas, conferem um benefício para a saúde no hospedeiro". Os mecanismos de ação dos probióticos incluem a acidificação da superfície mucosa, a prevenção da aderência de agentes patogénicos, a produção de substâncias como vitaminas e imunomoduladores e a ação sinérgica com o sistema imunológico do hospedeiro. Os probióticos utilizados como tratamento não-convencional, que contêm Lactobacillus e estabilizam a microbiota vaginal, são frequentemente usados no tratamento e prevenção da candidíase vulvovaginal, como terapêutica isolada ou em suplementação ao tratamento convencional. ${ }^{4}$ Estes mostraram efeito antimicrobiano in vitro na presença de diferentes microrganismos, como a C. albicans, para além da capacidade de colonizar da microflora vaginal. ${ }^{3,5} \mathrm{~A}$ abordagem da CVV recor- rente é difícil e não apresenta nível de evidência consistente para recomendar o tratamento convencional e ainda menos para o tratamento não-convencional. ${ }^{3}$

O objetivo desta revisão é determinar, à luz da evidência atual, se os probióticos contribuem para prevenção e tratamento de mulheres com CVV.

\section{MÉTODOS}

Procedeu-se a uma pesquisa de normas de orientação clínica (NOC) nas bases de dados National Guideline Clearinghouse, Guidelines do NHS Britânico, Canadian Medical Association Practice Guidelines Infobase e de meta-análises (MA), revisões sistemáticas (RS) e ensaios clínicos aleatorizados (ECA) nas bases de dados The Cochrane Library, DARE, Bandolier e MEDLINE/PubMed. Foram incluídas publicações entre março de 2007 e março de 2017, nas línguas portuguesa e inglesa. Na pesquisa foram utilizados os termos MeSH candidiasis vulvovaginal e probiotics.

Segundo o modelo PICO, os critérios utilizados para a inclusão dos artigos nesta revisão foram: População Mulheres de idade igual ou superior a 18 anos com candidíase vulvovaginal; Intervenção - Prevenção e/ou tratamento com probiótico; Controlo - Não fazem prevenção e/ou tratamento com probiótico; Outcome - Resolução e/ou não recorrência da candidíase vulvovaginal.

Definiram-se como critérios de exclusão: doença sistémica (e.g., DM, VIH), imunodepressão, gravidez, idade inferior a 18 anos, terapêutica tópica ou sistémica concomitante ou sequencial.

Para a classificação dos níveis de evidência (NE) e atribuição da força de recomendação (FR) utilizou-se a escala Strength of Recommendation Taxonomy (SORT), da American Academy of Family Physicians.

A seleção dos artigos para revisão foi feita em conjunto pelos autores, que decidiram excluir artigos duplicados ou ensaios clínicos incluídos nas RS ou MA selecionadas. A leitura integral foi realizada e a avaliação da qualidade e NE dos artigos incluídos discutida pelos autores.

\section{RESULTADOS}

Da pesquisa efetuada obtiveram-se 22 artigos. Destes foram excluídos, numa primeira fase, nove artigos: seis por divergência do objetivo do trabalho, dois por incumprimento de critérios de inclusão/exclusão e um por não estar disponível, mesmo após contacto com o 


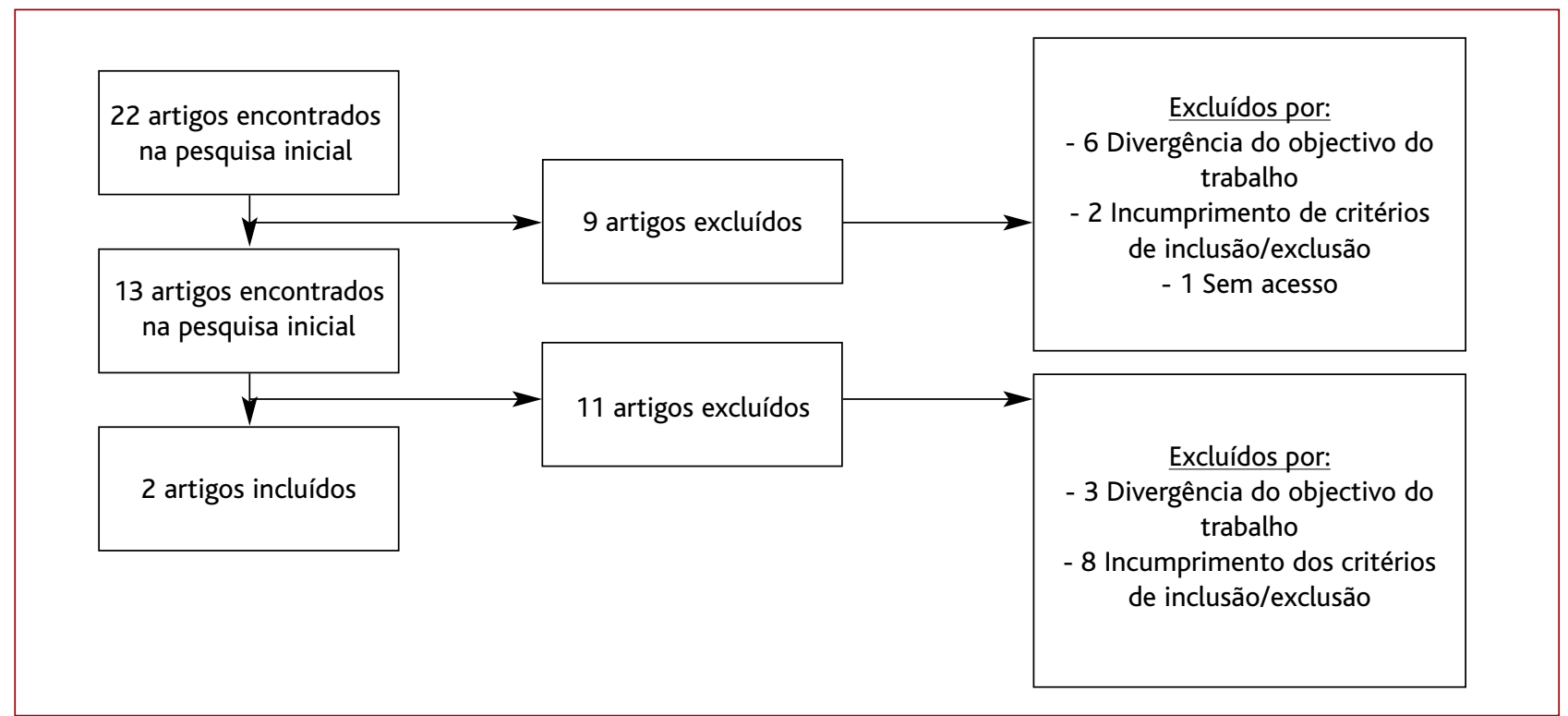

Figura 1. Organigrama da seleção dos artigos.

\section{QUADRO I. Tabela de resultados de Normas de Orientação Clínica}

\begin{tabular}{l|l|l|l|l} 
& \multicolumn{1}{|c|}{ Referência } & \multicolumn{1}{c|}{ Recomendações } & $\begin{array}{c}\text { Força de } \\
\text { Recomendação }\end{array}$ \\
\hline NOC & Mendling, et al (2012) & $\begin{array}{l}\text { Não está recomendado o uso de probióticos no tratamento e prevenção da } \\
\text { candidíase vulvovaginal }\end{array}$ & SORT A \\
\hline NOC & Lazaro, et al (2013) ${ }^{7}$ & $\begin{array}{l}\text { Evidência insuficiente para recomendar probióticos como tratamento } \\
\text { alternativo }\end{array}$ & SORT A
\end{tabular}

autor e local de publicação. Após leitura integral dos artigos restantes foram excluídos 11. Os motivos de exclusão foram, em três, por divergência do tema, sete por não cumprirem os critérios de inclusão/exclusão e uma RS em que, após leitura integral, dois dos seus ECA não cumpriam os critérios de inclusão e um dos seus ECA não estava acessível para leitura, mesmo após contacto com o autor e com o local de publicação.

Assim, foram incluídas duas NOC. O organigrama de seleção dos artigos está representado na Figura 1.

A NOC de Mendling e Brasch, ${ }^{6}$ de 2012, que incluiu sete ECA, três MA e duas NOC, não recomenda o uso de probióticos no tratamento e prevenção da CVV. A esta NOC foi atribuída força de recomendação A. A NOC da The British Association for Sexual Health and HIV, por Lazaro, $^{7}$ de 2013, refere que existe evidência insuficiente para, por rotina, recomendar o uso de probióticos, seja por via oral ou vaginal, como terapêutica alternativa da CVV. A esta NOC foi atribuída força de recomendação A. A Tabela 1 resume os resultados das NOC.

\section{CONCLUSÕES}

A CVV é uma patologia frequente, por isso se considera vantajosa a procura de alternativas terapêuticas, nomeadamente os probióticos.

Da análise dos artigos selecionados concluiu-se que não existe evidência do tratamento da CVV aguda nem na prevenção da CVV recorrente com probióticos (força de recomendação A).

A crescente resistência aos antibióticos poderá favorecer a futura investigação sobre a utilização dos probióticos no tratamento da CVV.

Foi uma limitação não poder aceder a todos os artigos encontrados na pesquisa, nomeadamente um arti- 
go de uma revisão sistemática que abordava a redução da incidência da CVV em utentes tratadas com probióticos isoladamente, e uma revisão sistemática que foi excluída por falta de acessibilidade a um dos ECA e outros dois ECA não cumprirem critérios de inclusão. A avaliação da qualidade dos artigos excluídos por falta de acesso fica, deste modo, condicionada.

Considera-se que são necessários mais estudos nesta área, de elevada qualidade, que suportem a evidência encontrada na formulação das recomendações.

\section{REFERÊNCIAS BIBLIOGRÁFICAS}

1. Watson $\mathrm{C}$, Calabretto H. Comprehensive review of conventional and non-conventional methods of management of recurrent vulvovaginal candidiasis. Aust N Z J Obstet Gynaecol. 2007;47(4):262-72.

2. Van Schalkwyk J, Yudin MH. Vulvovaginitis: screening for and management of trichomoniasis, vulvovaginal candidiasis, and bacterial vaginosis. J Obst Gynaecol Can. 2015;37(3):266-74.

3. Ehrström S, Daroczy K, Rylander E, Samuelsson C, Johannesson U, Anzén $B$, et al. Lactic acid bacteria colonization and clinical outcome after probiotic supplementation in conventional treated bacterial vaginosis and vulvovaginal candidiasis. Microbes Infect. 2010;12(10):691-9.

4. Bohbot JM, Cardot JM. Vaginal impact of the oral administration of total freeze-dried culture of LCR 35 in healthy women. Infect Dis Obstet Gynecol. 2012;2012:503648.
5. Kovachev SM, Vatcheva-Dobrevska RS. Local probiotic therapy for vaginal Candida albicans infections. Probiotics Antimicrob Proteins. 2015;7(1):38-44.

6. Mendling W, et al. Guideline vulvovaginal candidosis (2010) of the german society for gynecology and obstetrics, the working group for infections and infectimmunology in gynecology and obstetrics, the german society of dermatology, the board of german dermatologists and the german speaking mycological society. Mycoses. 2012; 55 Suppl 3:1-13.

7. Lazaro N. Sexually Transmitted Infections in Primary Care 2013 (RCGP/BASHH). Disponível em www.rcgp.org e www.bashh.org/guidelines

\section{CONFLITO DE INTERESSES}

Os autores declaram não possuir quaisquer conflitos de interesse.

\section{FINANCIAMENTO DO ESTUDO}

Os autores declaram que o trabalho relatado neste manuscrito não foi objeto de qualquer financiamento externo.

\section{ENDEREÇO PARA CORRESPONDÊNCIA \\ Lucélia Cristina Pedras Campinho \\ E-mail: lucelia_pedras@hotmail.com \\ https://orcid.org/0000-0003-0006-1738}

Recebido em 28-05-2017

Aceite para publicação em 02-09-2019

\section{ABSTRACT}

\section{PROBIOTICS IN WOMEN WITH VULVOVAGINAL CANDIDIASIS: WHAT IS THE EVIDENCE?}

Vulvovaginal candidiasis is one of the most common forms of female genital infection, affecting $75 \%$ of women at least once in their lifetime. It is associated with an imbalance of the vaginal microflora due to the consequent quantitative and/or qualitative alteration of the Lactobacillus and overgrowth of other vaginal mucosa commensals, namely Candida albicans. Probiotics, which contain Lactobacillus, are often used to treat and prevent vulvovaginal candidiasis.

The purpose of this review is to determine, in light of current evidence, whether probiotics contribute to the prevention and treatment of women with vulvovaginal candidiasis.

We conducted a survey of clinical guidelines, meta-analyses, systematic reviews, and randomized controlled trials published between March 2007 and March 2017 in Portuguese and English. The search used the MeSH terms 'Vulvovaginal Candidiasis' and 'Probiotics'. To assess the levels of evidence and assignment of strength of recommendation was used the Strength of Recommendation Taxonomy scale.

The search resulted in 22 articles, two of which were included. The rest were excluded because they did not meet the inclusion criteria, due to repetition or were not accessible.

The articles included are two clinical guidelines that concluded that the use of probiotics in the treatment and/or prevention of vulvovaginal candidiasis, both with A strength, is not recommended.

Further high-quality studies in this area are considered necessary to support the evidence found in the formulation of the recommendations.

Keywords: Candidiasis vulvovaginal; Probiotics. 